\title{
BMJ Open Identifying multimorbidity patterns of non-communicable diseases in paediatric inpatients: a cross-sectional study in Shanghai, China
}

Ning Chen, ${ }^{1}$ Liang Zhou, ${ }^{2}$ Jiaoling Huang (1),${ }^{2}$ Wenya $Y u,{ }^{2}$ Chen Chen, ${ }^{3}$ Hua Jin,,${ }^{4,5}$ Xiaoxiao Shi, ${ }^{4,5}$ Zhaohu Yu, ${ }^{6}$ Qian Liu, ${ }^{7}$ Yan Yang, ${ }^{7}$ Zhaoxin Wang (D) , 2,8 Jianwei Shi (i) 4,9

To cite: Chen N, Zhou L, Huang J, et al. Identifying multimorbidity patterns of non-communicable diseases in paediatric inpatients: a cross-sectional study in Shanghai, China. BMJ Open 2021;11:e042679. doi:10.1136/ bmjopen-2020-042679

- Prepublication history for this paper is available online. To view these files, please visit the journal online (http://dx.doi org/10.1136/bmjopen-2020042679).

$\mathrm{NC}$ and LZ contributed equally.

Received 11 July 2020 Revised 21 February 2021 Accepted 23 February 2021

Check for updates

(c) Author(s) (or their employer(s)) 2021. Re-use permitted under CC BY-NC. No commercial re-use. See rights and permissions. Published by BMJ.

For numbered affiliations see end of article.

\section{Correspondence to}

Dr Jianwei Shi;

shijianwei_amy@126.com and

Dr Zhaoxin Wang;

supercell002@sina.com

\section{ABSTRACT}

Objectives To enhance the understanding of noncommunicable disease (NCD) multimorbidity in children who are inpatients by delineating the characteristics of and identifying patterns among paediatric inpatients with multimorbidity in China.

Design Cross-sectional study.

Setting Paediatric wards $(n=17)$ in Pudong New Area, Shanghai, China.

Participants A total of 193432 paediatric inpatients in the electronic health record systems of 17 hospitals from 2011 to 2016 participated in the study, and 91004 children with NCDs were extracted and classified based on International Classification of Diseases, 10th version codes.

Main outcome measures Number of the NCDs and multimorbidity patterns of the paediatric inpatients. Results In total, $47.05 \%$ (95\% Cl 46.83 to 47.27 ) of the paediatric inpatients had one or more chronic diseases, and $16.30 \%(95 \% \mathrm{Cl} 16.14$ to 16.46$)$ had multimorbidity. Congenital anomalies accounted for $19.43 \%(95 \% \mathrm{Cl}$ 19.25 to 19.61) of the principal diagnoses among the paediatric inpatients. Five common multimorbidity patterns were identified: a neurological-respiratory cluster, a neurological-respiratory-ear cluster, a cardiovascularcirculatory cluster, a genitourinary cluster (boy group) and a musculoskeletal-connective cluster (10-18 years age group).

Conclusions Multimorbidity in paediatric inpatients suggests that decisions about reasonable allocation of paediatric inpatient resources should be fully considered. Multimorbidity patterns in paediatric inpatients revealed that prevention, including innovative treatments targeting children, should be further studied.

\section{INTRODUCTION}

Multimorbidity refers to the simultaneous existence of multiple chronic diseases or statuses in a single individual. ${ }^{1}$ Multimorbidity is thought to have a drastic and persistent influence not only on individuals but also on healthcare systems. ${ }^{2}$ On an individual level, people with multimorbidity often suffer from
Strengths and limitations of this study

- This study is one of the few studies that focused on the multimorbidity patterns, particularly in hospitalised children of different age groups and sex.

- A relatively more accurate analysis of the multimorbidity patterns based on our subjects could be attributed to the inclusion of all chronic conditions registered in electronic health record.

- The diagnosis data can avoid recall bias, which is inevitable in self-reported data derived from surveybased studies.

- The data of the inpatients in this study were limited to serious cases because they were inpatients, and additional data of outpatients should be included in further analyses.

- The data of paediatric inpatients in this study might help to reveal the situation of multimorbidity situation existing in children and youth, because the multimorbidity conditions of children and youth are usually more common in inpatients than outpatients.

adverse drug events, ${ }^{3}$ poor functional status, ${ }^{4}$ negative occupational consequences, ${ }^{5}$ poor health-related quality of life, ${ }^{6}$ prolonged or frequent hospitalisations ${ }^{2}$ and even increased risks of disability and mortality. ${ }^{7}$ From a macroscopic point of view, the complications of medical treatment processes will decrease the efficiency of the healthcare system. Furthermore, because of the long-lasting and repetitive characteristics of multimorbidity, repeat hospitalisation and high health service utilisation ${ }^{9}$ by individuals will consume scarce medical and health resources and enhance the economic burden of the medical system. ${ }^{10}$

Nearly half of the previous studies on multimorbidity have focused on elderly populations. ${ }^{11}$ Evidence in many parts of the world has indicated that an extensive and probably increasing proportion of the adult population has multiple chronic health conditions. ${ }^{12}$ 
The prevalence of multimorbidity has been estimated to be approximately $50 \%$ among people aged younger than 65 years, $62 \%$ among those aged between 65 and 74 years, and $81.5 \%$ among those aged older 85 years, increasing gradually with age. ${ }^{4}$ Although multimorbidity has been regarded as one of the essential health concerns of the middle-aged and older population, studies have found that a considerable number of young populations also face the threat of multimorbidity. ${ }^{2}$ Worse, earlyonset multimorbidity has the potential to complicate and even worsen health conditions, because it lengthens and compounds the time spent with a poor health status with age. ${ }^{10}$ Ferro et $a l^{13}$ focused on multimorbidity in a population with mental health conditions and analysed how multimorbidity in childhood and youth influences interactions with the healthcare system based on a sample of 250 children. Dunbar et $a l \mathrm{~s}^{14}$ study assessed variations across complex chronic diseases and the likelihood of readmission with increasing age in adolescents and young adults. However, few quantitative studies have focused on the overall multimorbidity status of children. ${ }^{15}$ The applicability of multimorbidity patterns in paediatric research and clinical practice requires further knowledge of the proportion, chronic diseases involved, their relationship with different paediatric age groups, and the existence of potential gender differences.

This study aimed to understand non-communicable disease (NCD) multimorbidity in children who are inpatients by delineating their characteristics and identifying patterns in paediatric inpatients living with multimorbidity in China. Specifically, the multimorbidity patterns in paediatric inpatients based on electronic health records (EHRs) by age and gender groups were identified in this study. We believe that understanding the coexistence patterns of chronic diseases among paediatric inpatients will help elucidate implications for the better prevention and treatment of multimorbidity as well as guide the suitable allocation of health resources among this population.

\section{METHODS}

\section{Study population}

A cross-sectional analysis was performed in Shanghai, China, a metropolitan city with a population of approximately 24.20 million, through the end of $2016 .{ }^{16}$ We extracted the data of paediatric inpatients with NCDs from the EHR systems of 17 hospitals from 2011 to 2016 in Pudong New Area, Shanghai, the largest district in Shanghai, comprising both urban and suburban areas. ${ }^{17}$ By the end of 2016, the area comprised approximately 5.50 million people $(22.73 \%$ of the total population of Shanghai) (Shanghai Statistics Bureau 2018). ${ }^{18}$ The registered population aged 17 years and younger was approximately 0.38 million in Pudong New Area at the end of 2016, accounting for $12.9 \%$ of all the age groups. The number of hospitals in the district is 25 . We included all
17 hospitals with paediatric wards, and all the hospitals included were public hospitals.

\section{Data collection}

The retrospective data (2011-2016) of hospitalisations on 193432 inpatients aged $0-18$ years were extracted from the EHR systems of all (17) hospitals with paediatric wards in the Pudong district of Shanghai. Not all the children who were admitted to the hospital had at least an overnight stay. Among all the paediatric inpatients, 1874 $(0.97 \%)$ paediatric inpatients did not stay overnight. For repeat visits in this database, only information related to the first admission was collected. The data of 91004 children with NCDs were extracted and classified into various groups according to their principal diagnosis based on International Classification of Diseases, 10th version (ICD-10) codes. The final dataset comprised the basic demographic information (sex and age), presence and patterns of chronic diseases (disease systems, disease categories, and multimorbidity status).

\section{Variables}

Disease categories and various diseases were coded and classified in accordance with the ICD-10. The EHR of the inpatients contains basic information of the inpatients, up to 11 disease diagnoses, and other information related to hospitalisation. All the diagnoses and disorders were identified by clinicians. The main disorder was determined according to the professional judgement of the doctor and adhered to the following principles: (1) the aetiological diagnosis includes the clinical manifestation of the disease; (2) if the purpose of hospitalisation was for surgical treatment, the disease consistent with surgical treatment is selected as the principal diagnosis; (3) if the patient is admitted to the hospital with a suspected diagnosis but is still undiagnosed at the time of discharge, the diagnosis of the disease with a high degree of clinical suspicion and greatest tendency is selected as the main diagnosis; (4) if a patient is admitted to the hospital because of an abnormal symptom, sign or examination result, and the diagnosis is unclear at discharge, the principal diagnosis should be the result of the symptom, sign or abnormal examination result; (5) if the disease manifests clinically as different degrees of harm during the occurrence and development of the disease, and hospitalisation is to diagnose and treat certain clinical manifestations, then the clinical manifestations are selected as the principal diagnosis; (6) when the hospitalisation is for the treatment of a complication of a disease, the complication is treated as the principal diagnosis. Chronic conditions were identified using the Healthcare Cost and Utilization Project Chronic Condition Indicator (HCUP-CCI) tool. ${ }^{19}$ The HCUP-CCI tool is a listing of all ICD-10-Clinical Modification codes classified as 'chronic' or 'nonchronic' with chronic conditions defined as conditions lasting 12 months or longer resulting in functional or social limitations and/or the need for ongoing medical care. Chronic diseases were first recognised according 
to ICD-10, and then multimorbidity patterns were determined based on the data of the paediatric inpatients and expertise of physicians. Patterns in this study equalled combinations, meaning that all the diseases appeared in each paediatric inpatient during this hospitalisation. We analysed the multimorbidity patterns and how they were distributed according to social demographics (sex and age) and clinical variables (multimorbidity status and number of chronic diseases). The inpatients were divided into three groups according to growth stage: $0-4$ years age group (infant to toddler age), 5-9 years age group (preschool age and early school age), 10-18 years age group (adolescence) $)^{20.21}$

\section{Data analysis}

Basic descriptive statistics were used to depict the personal characteristics of the paediatric inpatients (including sex, age and principal diagnosis for hospitalisation), and the denominator of the proportions in this part was all the 193432 paediatric inpatients. All the paediatric inpatients were included irrespective of whether they were diagnosed with multiple chronic diseases. The rankings of the NCD groups and disorders in different sex and age groups were sequenced according to their relative proportions, and the denominator was the patients whose principal diagnosis was NCD. Additionally, 95\% CIs for proportions were calculated using the normal approximation to binomial distributions. All the data were analysed using SPSS V.21.0.

\section{Patient and public involvement}

No patients or public were involved in the design, outcome measures, recruitment to or conduct of this study. Given the nature of removing all individual information, there is no requirement to disseminate the information to patients.

\section{RESULTS}

\section{Personal characteristics of paediatric inpatients with NCDs}

In total, 193432 paediatric inpatients with chronic diseases were identified between 2011 and 2016 (table 1). Most of the patients were boys $(62.73 \%)$, and most were $0-4$ years old (58.93\%). Additionally, 31522 (16.30\%; 95\% CI 16.14 to 16.46) met the criteria of multimorbidity. Most of the inpatients with multimorbidity had two chronic diseases $(\mathrm{n}=19360 ; 61.42 \%)$. Among all the registered population under 18 years in the Pudong district of Shanghai (2016), the proportion of the paediatric inpatients with multimorbidity within 6 years (2011-2016) was $8.29 \%$ (95\% CI 8.20 to 8.37$)$.

Ranking of the NCD Disease Groups according to sex and age Regarding the principal diagnoses, 91004 (47.05\%, 95\% CI 46.83 to 47.27) paediatric inpatients had an NCD as their main disorder. The NCDs were classified according to the relevant disease patterns, and the classified NCD Disease Groups (NCD Disease systems) were ranked according to the proportions by sex (table 2). Remarkably, congenital anomalies, deformations and chromosomal abnormalities ranked first in both boys $(39.20 \%)$ and girls $(45.26 \%)$ and accounted for $19.43 \%$ (37 585/193 432, 95\% CI $19.25 \%$ to $19.61 \%$ ) of the main diagnosis of paediatric inpatients who had an NCD as their main disorder. Diseases of the genitourinary system ranked far higher in boys $(11.20 \%$, third rank) than in girls (4.08\%, eighth rank). Moreover, the rank of diseases of the digestive system (boys, $5.66 \%$, sixth rank; girls, $4.58 \%$, seventh rank) in boys was slightly higher than that in girls. Compared with those in boys, the proportions of diseases of the circulatory (boys, $5.43 \%$, seventh rank; girls, $6.09 \%$, fifth rank) and musculoskeletal systems and connective tissue (boys, $4.12 \%$, eighth rank; girls, $4.84 \%$, sixth rank) were higher in girls. For both sexes, congenital anomalies, deformations and chromosomal abnormalities (boys, $39.20 \%$, first rank; girls, $45.26 \%$, first

Table 1 Demographic characteristics of paediatric inpatients in 2011-2016 ( $n=193432)$

\begin{tabular}{|c|c|c|c|c|}
\hline Variable & Classification & $\begin{array}{l}\text { Multimorbidity (n, (\%, } \\
95 \% \text { Cl) (n=31522) }\end{array}$ & $\begin{array}{l}\text { Non-multimorbidity ( } \mathrm{n},(\% \text {, } \\
95 \% \mathrm{CI}))(\mathrm{n}=161910)\end{array}$ & $\begin{array}{l}\text { All }(n,(\%, 95 \% C l)) \\
n=(193432)\end{array}$ \\
\hline \multirow[t]{2}{*}{ Gender } & Boy & $19793(62.79,62.26$ to 63.32$)$ & $101545(62.72,62.48$ to 62.96$)$ & $\begin{array}{l}121338(62.73,62.51 \text { to } \\
62.95)\end{array}$ \\
\hline & Girl & $11729(37.21,36.68$ to 37.74$)$ & $60365(37.28,37.04$ to 37.52$)$ & $72094(37.27,37.05$ to 37.49$)$ \\
\hline \multirow[t]{2}{*}{ Age (year) } & $0-4$ & $20836(66.10,65.58$ to 66.62$)$ & $93160(57.54,57.30$ to 57.78$)$ & $\begin{array}{l}113996(58.93,58.71 \text { to } \\
59.15)\end{array}$ \\
\hline & $10-18$ & $3969(12.59,12.22$ to 12.96$)$ & $29347(18.13,17.94$ to 18.32$)$ & $33316(17.22,17.05$ to 17.39$)$ \\
\hline \multirow{3}{*}{$\begin{array}{l}\text { Number } \\
\text { of chronic } \\
\text { diseases }\end{array}$} & 0 & $0(0.00)$ & $86891(53.67,53.43$ to 53.91$)$ & $86891(44.92,44.70$ to 45.14$)$ \\
\hline & 1 & $0(0.00)$ & $75019(46.33,46.09$ to 46.57$)$ & $75019(38.78,38.56$ to 39.00$)$ \\
\hline & 2 & $19360(61.42,60.88$ to 61.96$)$ & $0(0.00)$ & $19360(10.01,9.88$ to 10.14$)$ \\
\hline
\end{tabular}




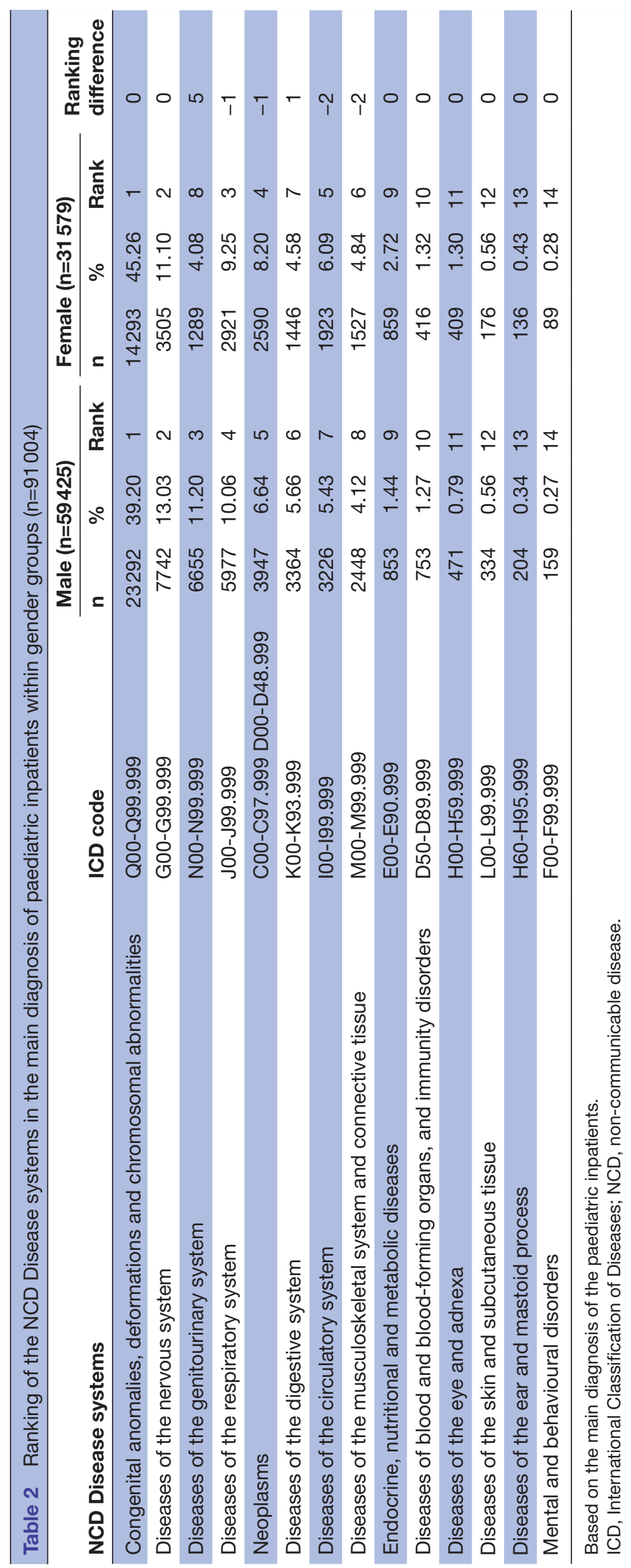


rank) and diseases of the nervous system (boys, 13.03\%, second rank; girls, $11.10 \%$, second rank) accounted for more than half of the main diagnosis of paediatric inpatients who had an NCD as their main disorder. Furthermore, diseases of the respiratory system ranked in the top five most frequent NCDs in paediatric inpatients (boys, $10.06 \%$, fourth rank; girls, $9.25 \%$, third rank). Notably, neoplasms ranked fourth in girls $(8.20 \%)$ and fifth in boys $(6.64 \%)$.

The ranks of the most frequent diseases across different age groups $(0-4,5-9,10-18$ years) are displayed in table 3. Congenital anomalies, deformations and chromosomal abnormalities were the most frequent NCDs in all age groups in this study $(0-4,51.75 \%$, first rank; $5-9$, $27.35 \%$, first rank; $10-18,21.70 \%$, first rank) $(\mathrm{p}<0.001)$, while the proportions decreased with age. The ranks of diseases of the respiratory system $(0-4,11.09 \%$, second rank; 5-9, 8.16\%, fourth rank; $10-18,7.12 \%$, eighth rank) $(\mathrm{p}<0.001)$ and diseases of the nervous system $(0-4$, $9.21 \%$, third rank; $5-9,23.10 \%$, second rank; $10-18$, $8.22 \%$, sixth rank $)(\mathrm{p}<0.001)$ were relatively higher in the $0-4$ and $5-9$ years age groups than in the 10-18 years age group. Notably, across all age groups, neoplasms were in the top five NCDs (0-4 years, $5.80 \%$, fourth rank; 5-9 years, $8.08 \%$, fifth rank; $10-18$ years, $11.24 \%$, third rank) $(\mathrm{p}<0.001)$. In addition to neoplasms, diseases of the genitourinary system $(0-4$ years, $5.50 \%$, fifth rank; $5-9$ years, $11.79 \%$, third rank; $10-18$ years, $16.70 \%$, second rank) $(p<0.001)$ were also in the top five most frequent NCDs, with the proportions increasing with age. Diseases of the circulatory system were more common in the 10-18 years (8.93\%, fourth rank) age group than in the $0-4$ years (4.83\%, seventh rank) and 5-9 years (5.64\%, sixth rank) age groups $(\mathrm{p}<0.001)$.

\section{Comparison of the multimorbidity patterns among the different sex and age groups}

In addition to those without multimorbidity or without NCDs, the most frequently occurring pattern was the coexistence of the two NCDs in both boys $(10.29 \%)$ and girls $(9.53 \%)$ (figure 1). The number of paediatric inpatients with two NCDs was greater than number of those with more than two NCDs (figure 1). Notably, the percentage of patients with multimorbidity in the $0-4$ years age group $(18.28 \%)$ was greater than that in the $5-9$ years $(14.56 \%)$ and 10-18 years age groups (11.91\%). Additionally, among those with the same number of NCDs $(n=2,3,4$, $\geq 5$ ), the percentages in the $0-4$ and 5-9 years age group were higher than those in the 10-18 years age group.

Tables 4 and 5 demonstrate the patterns of multimorbidity among the different sex and age groups. Multimorbidity comprising sleep apnoea and chronic rhinitis was the second most frequent multimorbidity pattern in both boys and girls in the two-disorders group (1057, 8.19\%; $504,8.77 \%$ ), we called this the neurological-respiratory cluster. The combination of sleep apnoea and allergic rhinitis was the third most frequent $(796,6.17 \%$; 376, $6.54 \%)$. The cardiovascular-circulatory multimorbidity pattern was common in both the boy and girl groups. Ventricular septal defects (VSDs) and atrial septal defects (ASDs) were the most frequently co-occurring morbidities in girls $(598,10.41 \%)$. Notably, the genitourinary cluster, comprising several disorders, was present in boys but not in girls (table 4). Congenital malformations of the penis together with redundant prepuce, phimosis or paraphimosis were the most frequently co-occurring morbidities in boys $(1546,11.98 \%)$. The patterns (neurological-respiratory-ear cluster) of sleep apnoea, chronic rhinitis and other chronic non-suppurative otitis media (295, 6.38\%, first rank; 148, 4.93\%, second rank); sleep apnoea, allergic rhinitis and other chronic nonsuppurative otitis media (221, 4.78\%, second rank; 106, $3.53 \%$, third rank); and VSD, ASD and other secondary pulmonary hypertension $(218,4.71 \%$, third rank; 179 , $5.96 \%$, first rank) were the top three multimorbidity patterns in both boys and girls. The cardiovascularcirculatory cluster, comprising congenital malformations, deformations and chromosomal abnormalities, combined with circulatory system disorders or congenital malformations, deformations and chromosomal abnormalities alone, were the most frequent multimorbidity patterns involving four NCDs in both sexes. In addition to the cardiovascular-circulatory cluster, the neurological-respiratory-ear cluster was also apparent in the four NCD compositions.

In the different age groups, the multimorbidity pattern (musculoskeletal-connective cluster) of two NCDs, systemic lupus erythematosus (SLE) and SLE with organ or system involvement, was more frequent in patients aged 10-18 years who had two NCDs (82, $2.73 \%$, second) and three NCDs $(10,1.49 \%$, second) than in patients aged $0-4$ and 5-9 years. In terms of the multimorbidity pattern of three NCDs, VSD, ASD and other secondary pulmonary hypertension were the most frequently occurring conditions in the $0-4$ years age group $(386,6.89 \%)$. Among those with three NCDs in the neurological-respiratory-ear cluster, sleep apnoea, chronic rhinitis and other chronic non-suppurative otitis media showed a high incidence in the $0-4$ years $(237,4.23 \%$, second) and $5-9$ years (202, $14.97 \%$, first) age groups but a relatively low incidence in the $10-18$ years age group $(8,1.19 \%$, fourth). Among those with three NCDs in the neurological-respiratory cluster, the incidence of sleep apnoea, chronic rhinitis and chronic tonsillitis $(124,9.19 \%$, third; 10, 1.49\%, third) and sleep apnoea, chronic tonsillitis and allergic rhinitis $(116,8.60 \%$, fourth; $19,2.84 \%$, first) were higher in the 5-9 and 10-18 years age groups than in the $0-4$ years age group. The cardiovascular-circulatory cluster comprising Tetralogy of Fallot, atrial septal defects and patent ductus arteriosus (PDA) was the fifth most frequent NCD cluster in the $0-4$ years age group, but it was not in the top five clusters in the 5-9 and 10-18 years age groups with three NCDs. Furthermore, the combination of epilepsy, other reduction deformities of the brain and primary adrenocortical insufficiency, with or without thrombocytopenia, were apparent in the $10-18$ years age 


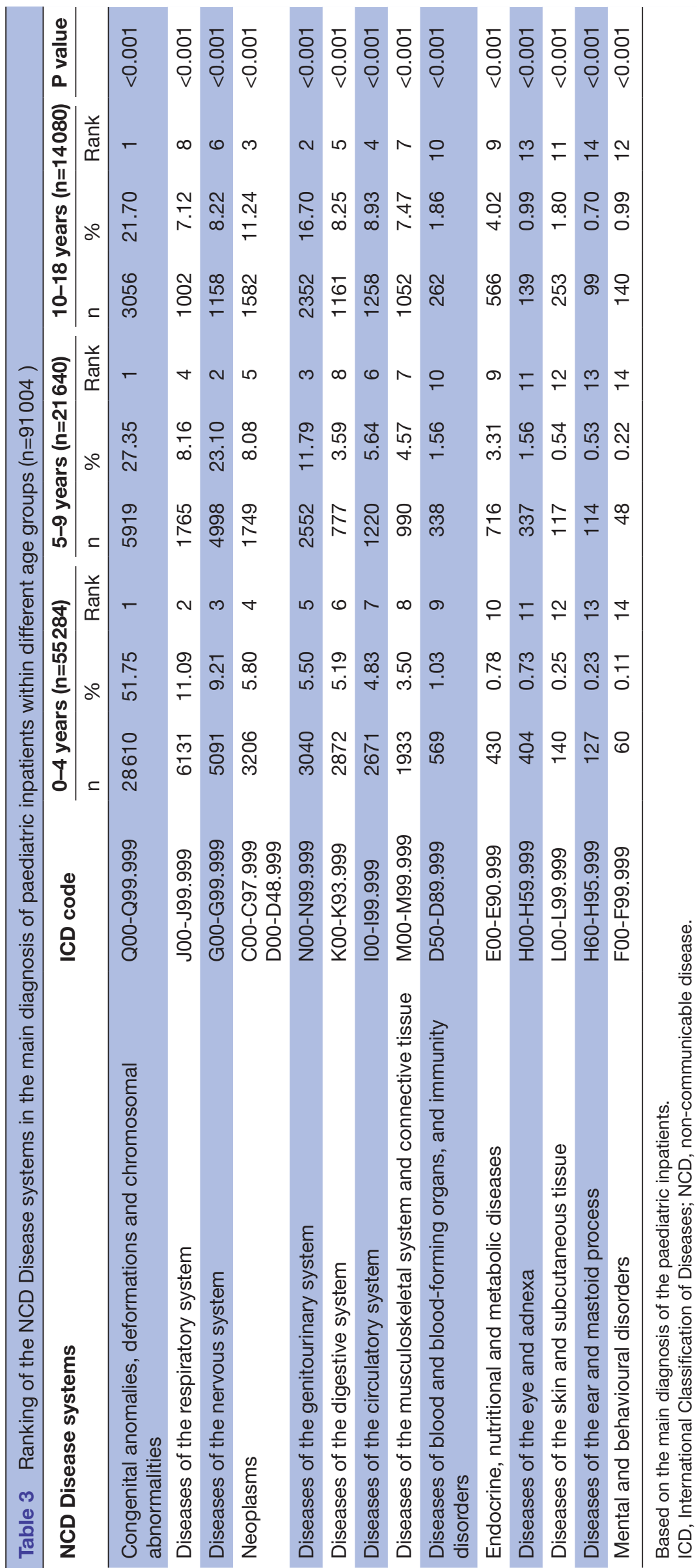




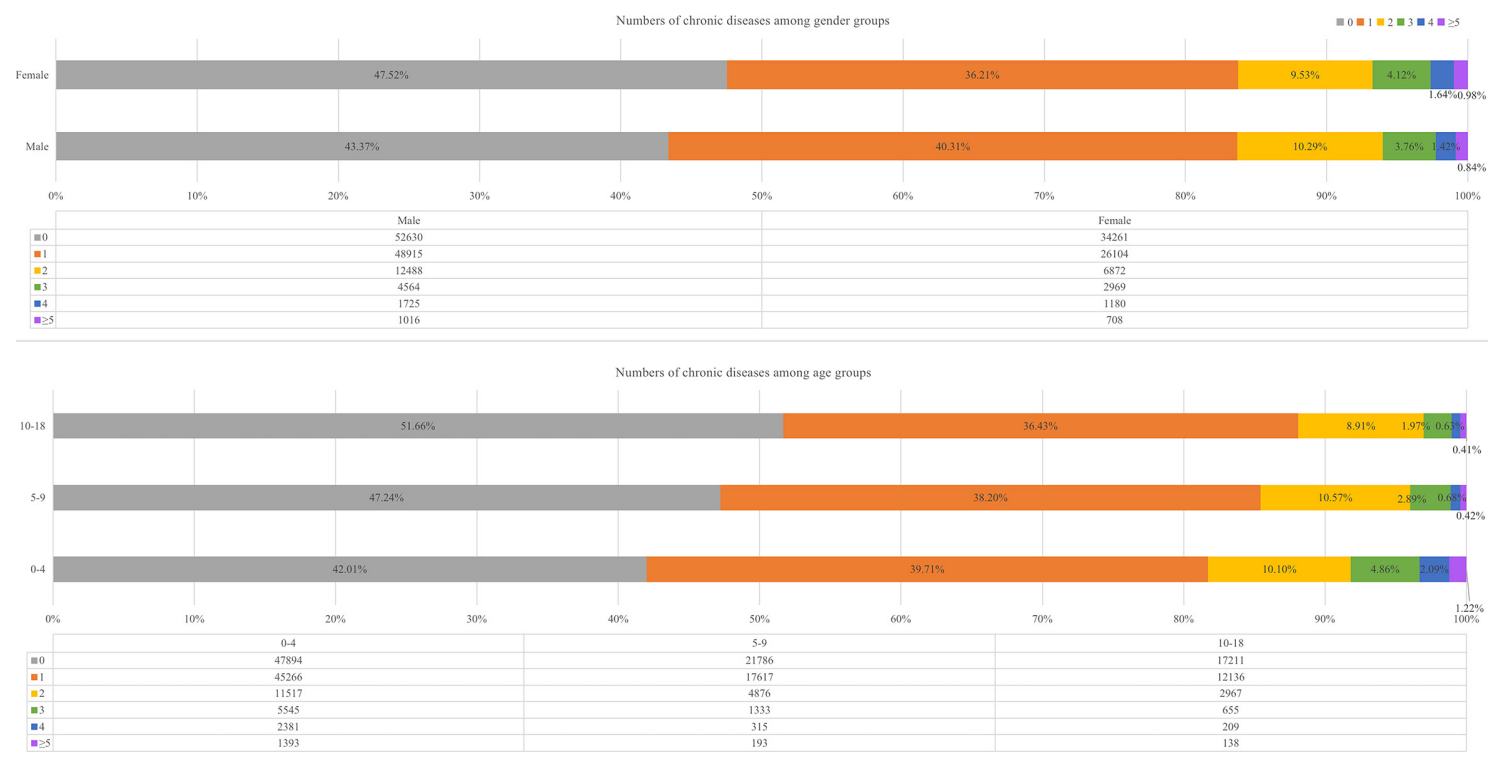

Figure 1 Numbers of chronic diseases among gender groups and age groups. Grey represents the paediatric inpatients with no NCDs. Orange represents the paediatric inpatients with one NCD. Yellow represents the paediatric inpatients with two NCDs. Green represents the paediatric inpatients with three NCDs. Blue represents the paediatric inpatients with four NCDs. Purple represents the paediatric inpatients with more than five NCDs. NCD, non-communicable disease.

group. The cardiovascular-circulatory cluster comprised most of the patterns of the four NCDs (table 5).

\section{DISCUSSION}

Multimorbidity is present in children and youth as well as adults and constitutes a challenge for individuals, families and the healthcare system. In our study, $16.30 \%$ of paediatric inpatients aged $0-18$ years had multimorbidity. This finding differed from that reported in previous studies in which the prevalence of multimorbidity among children was lower than $10 \%$. For example, in Karen's study, regardless of the socioeconomic status, the prevalence of multimorbidity in people younger than 18 years was lower than $4.0 \%$ among all registered patients from 314 medical practices caring for about one-third of the Scottish population. ${ }^{22}$ Additionally, findings from a large New Zealand child cohort reported multimorbidity in $9.7 \%$ of the cohort children. ${ }^{23}$ The disparities might be attributed to diverse data sources because the subjects in this study were paediatric inpatients whose conditions were comparatively more serious than paediatric outpatients.

In our study, congenital anomalies, deformations and chromosomal abnormalities accounted for the highest proportion $(19.43 \%)$ of NCDs; in the $0-4$ years age group, congenital anomalies accounted for most $(14.79 \%)$ NCDs. However, in one study in the USA, congenital anomalies were present in approximately $3 \%$ of all live births in the USA, ${ }^{24}$ and they were present in 199 per 10000 children in Addis Ababa and the Amhara region of Ethiopia. ${ }^{25}$ Globally, 6\% (8.1 million) of newborn children have congenital anomalies of genetic or partial genetic origins. ${ }^{26}$ This difference may be due to our data being extracted from the EHRs of paediatric inpatients whose conditions were often worse than those of outpatients; moreover, we included a broader age spectrum than previous studies on newborns. ${ }^{26}$ Among all the diseases classified as congenital anomalies, deformations and chromosomal abnormalities, cardiovascular abnormalities were perceived to appear more frequently than other abnormalities in this study. This finding agrees with the findings of Dolk et al, who discerned that congenital heart defects (CHDs) were the most common nonchromosomal conditions, at 6.5 per 1000 births, among 1.5 million annual births in 22 countries in a common protocol and data quality review. ${ }^{2728}$ Musculoskeletal anomalies were shown to be the most common (more than half) congenital anomalies in a 4-year prospective study. ${ }^{29}$ Preventive measures for congenital anomalies in children should be adopted according to the type or cluster of anomalies; these measures may depend on further investigation of risk factors for the corresponding anomalies.

Five multimorbidity patterns were discerned in this study. The first (neurological-respiratory cluster) incorporates neurological and respiratory disorders. The main diseases included sleep apnoea, sleep disorders, chronic rhinitis, allergic rhinitis and adenoidal hypertrophy. All the conditions listed above were found in this pattern. A previous review noted that one of the dominating comorbidities associated with allergic rhinitis was sleep disturbance, which was consistent with the pattern we proposed. ${ }^{30}$ A retrospective cross-sectional study among 146 children aged 2-12 years showed that chronic rhinitis was present in $43 \%$ of children with obstructive sleep apnoea (OSA), indicating that rhinitis is an important comorbidity in children with OSA. ${ }^{31}$ Adenoidal hypertrophy together with disturbances in nasal respiration and tonsillar hypertrophy is a risk factor for OSA in primary school children. ${ }^{33}$ A more complicated pattern 


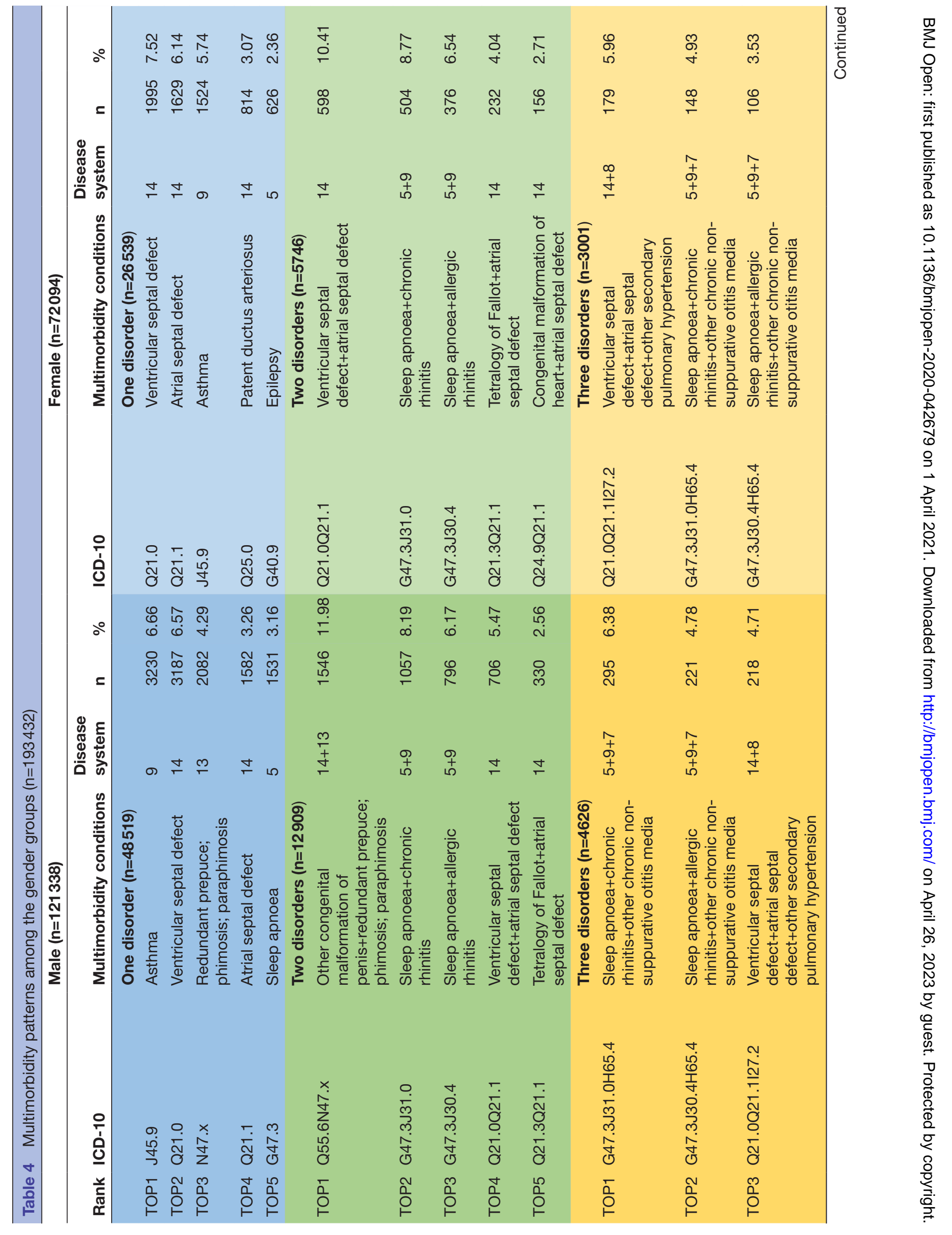




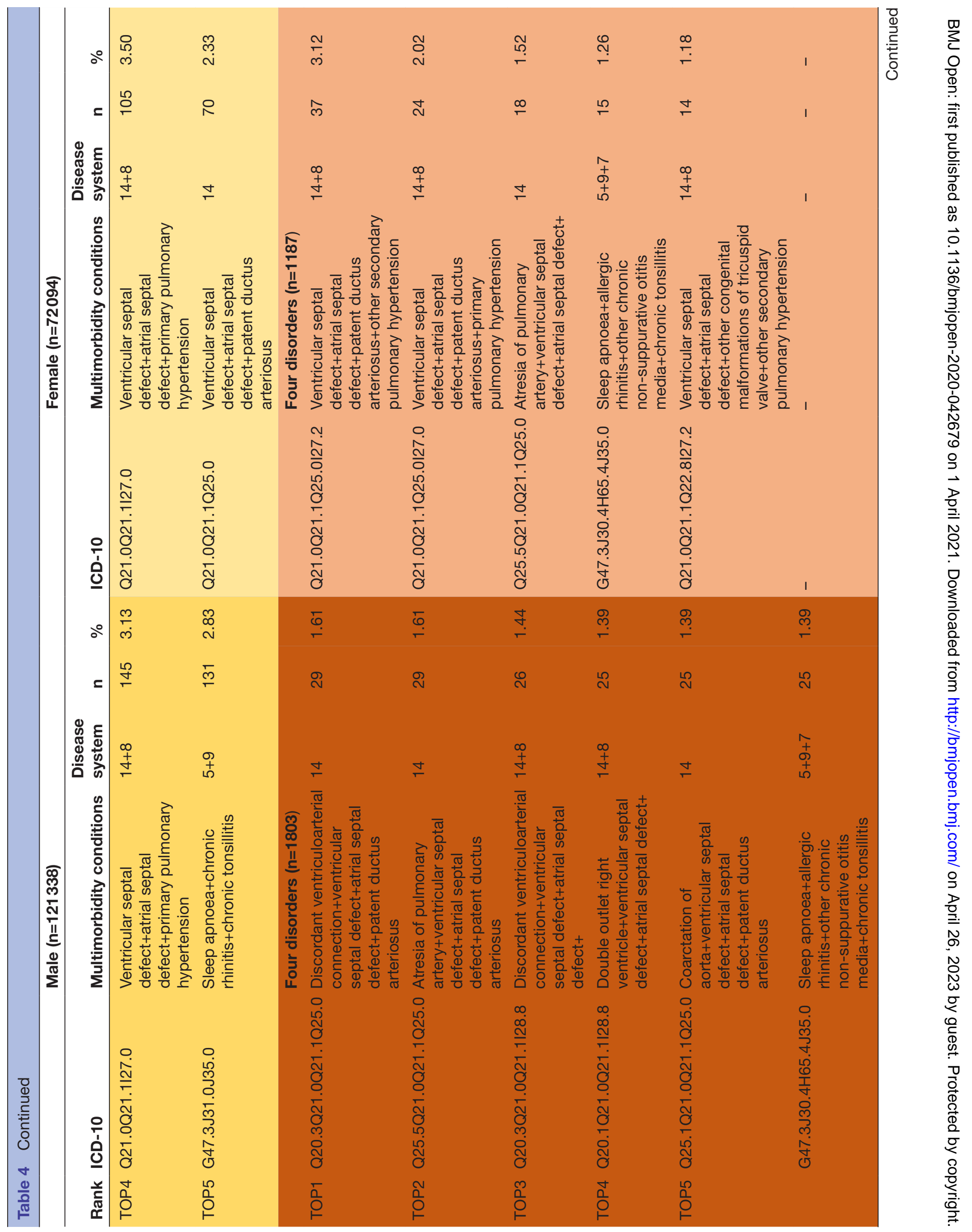




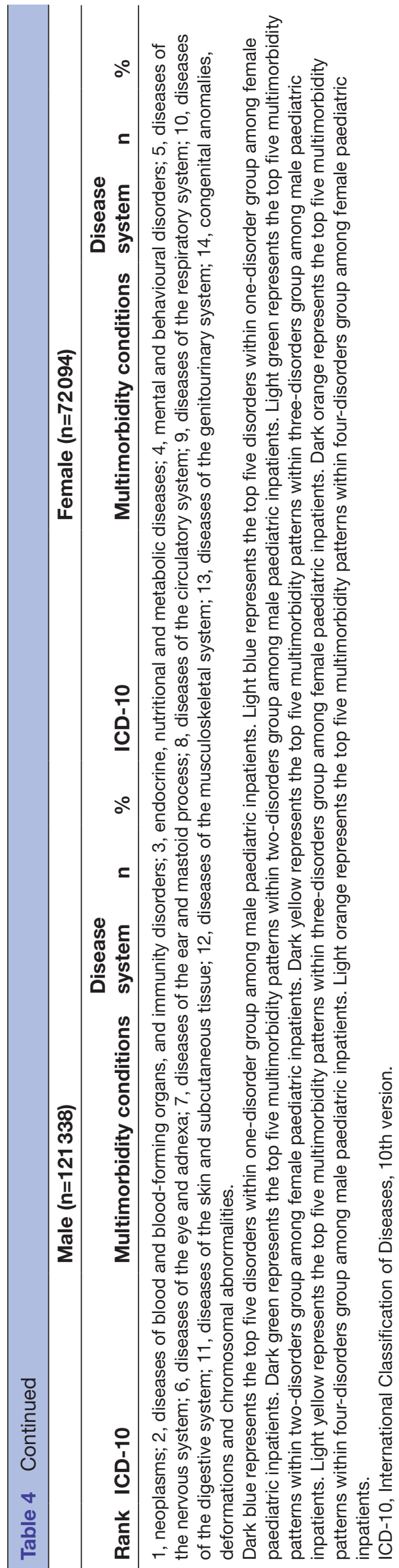

(neurological-respiratory-ear cluster) was found within the neurological-respiratory cluster with other chronic nonsuppurative otitis media. The chronic impacts of the inflammatory process affect other relevant systems or organs-for example, the ears, lungs and others. In the development of chronic otitis media, rhinitis may be linked through two mechanisms: a decrease in the ciliary beat frequency or eustachian tube dysfunction caused by allergic reactions in the nasal mucosa. ${ }^{34}$ Considering that this cluster had the highest frequency in children and adolescents, the comorbidities associated with allergic rhinitis, such as OSA syndrome, otitis media and aggravation of adenoidal hypertrophy, ${ }^{29}$ should be given special attention when developing relative clinical care guidelines for young populations.

Another common pattern (cardiovascular-circulatory cluster) comprised VSD, ASD, other secondary pulmonary arterial hypertension, pulmonary atresia, PDA and primary pulmonary arterial hypertension. In line with our pattern, VSD and ASD were the most common CHDs reported in other studies. ${ }^{35}{ }^{36}$ Additionally, diverse variations were found in the corresponding frequencies of other CHDs. ${ }^{37}$ As a common complication of CHD, pulmonary arterial hypertension associated with CHD (PAH-CHD) is the second most common type of $\mathrm{PAH}$ in children, accounting for more than $40 \%$ of cases, particularly in developing and undeveloped areas. ${ }^{38}{ }^{39}$ Because of apparent disparities between the management of paediatric PAH-CHD and adult PAH-CHD, ${ }^{40}$ guidelines for paediatric $\mathrm{PAH}$-specific therapies extrapolated from clinical experience and adult clinical trials ${ }^{41} 42$ are not well suited for children. ${ }^{43}$

Notably, the compositions of the patterns between the different sexes in the paediatric inpatients were similar. However, a specific pattern (genitourinary cluster) in boys must be mentioned. Although not mentioned in other studies, the composition of other congenital malformations of the penis and redundant prepuce, phimosis or incarcerated phimosis was present in a high proportion of boys in this study; this result deserves additional attention in future investigations.

Notable results arose when comparing the patterns of multimorbidity across different age groups of paediatric inpatients. One cluster (musculoskeletal-connective cluster), which mainly comprised musculoskeletal and connective tissue disorders, was notable in the 10-18 years age group. Groups of symptoms depicted in this pattern have appeared mainly under the labels of SLE and SLE with organ or system involvement. As a chronic, multisystem autoimmune disease, childhood-onset SLE begins before 18 years of age and can harm any organ system; it has a broad scope of disease manifestations, which could result in remarkable morbidity and even mortality. ${ }^{44}$ For example, clinical features involving glomerulonephritis and the central nervous system have been implicated more often in children with SLE than in adults. ${ }^{45}$ A study on the comorbidity index in children with SLE also indicated that it is not uncommon for the initial manifestations of comorbid lesions in organs and other systems to occur in children and adolescents, potentially leading to continuous morphological and functional disorders, morbidity and 


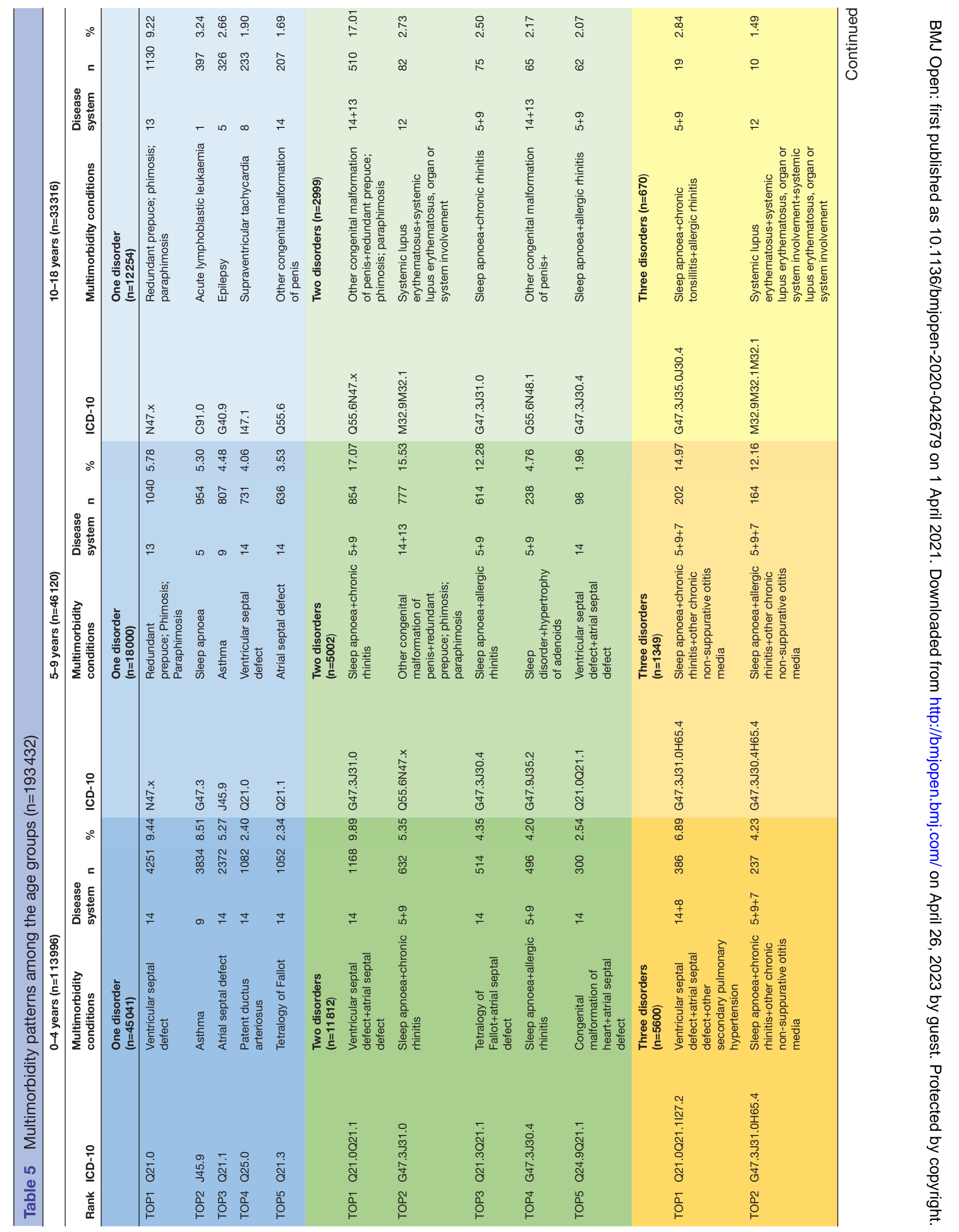




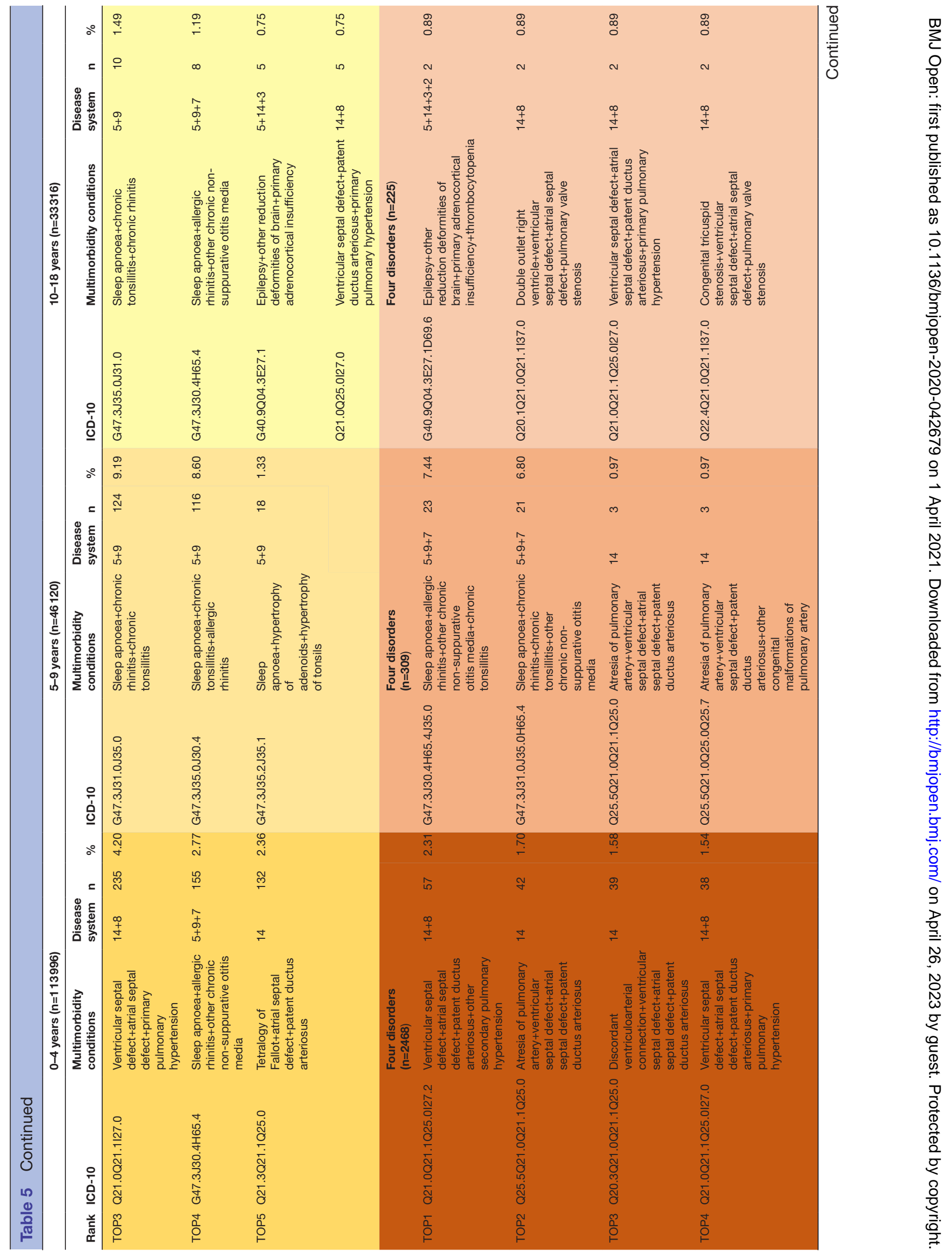



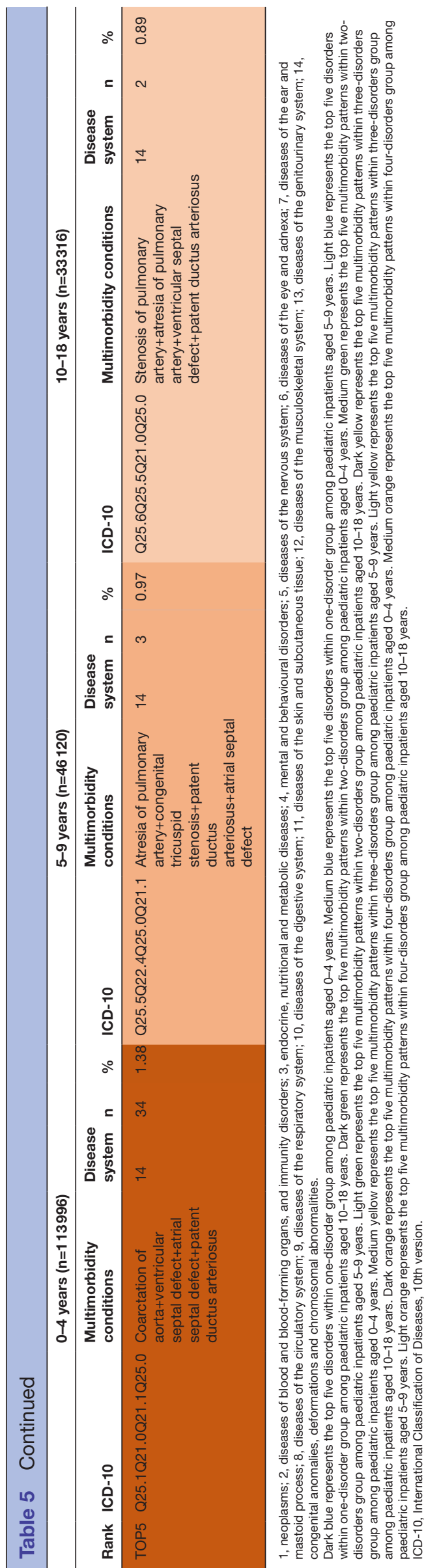

mortality in children. The underlying mechanism responsible for developing the above comorbid conditions might be associated with the disruption of lipid metabolism and blood coagulation ${ }^{46}$ and deserves further confirmation in future studies.

This study possesses several strengths that deserve to be mentioned. The study was based on medical diagnosis information extracted from EHRs, suggesting that the data were accurate. ${ }^{47}$ Furthermore, attributed to the data source extracted from EHRs, the recall bias which is inevitable in self-reported data derived from survey-based studies is avoided in this study. As one of the few studies focused on the multimorbidity patterns of hospitalised children with different age groups and sex, the findings of our study could increase the understanding of the multimorbidity in children to some extent. However, several limitations must be considered. First, the data of the inpatients were limited to serious cases because they were inpatients; additional data of outpatients should be included in further analyses. Second, the data of paediatric inpatients might produce an over estimate of the multimorbidity prevalence in children and youth because most of the inpatients had serious health conditions. Third, the sample was from Pudong New Area in Shanghai, China, and the survey results may not represent other areas. The study should be extended to include a larger sample of paediatric hospitals in additional regions.

\section{CONCLUSIONS}

The neurological-respiratory cluster, neurologicalrespiratory-ear cluster and cardiovascular-circulatory cluster were common in various sex and age groups among paediatric inpatients in this study. A thorough understanding of the multimorbidity patterns may facilitate the screening and identification of specific conditions coexisting with chronic diseases in the young population. Further studies are still needed concerning prevention including innovative treatments targeting children with common multimorbidity patterns.

\section{Author affiliations}

${ }^{1}$ Tongji University School of Medicine, Shanghai, China

${ }^{2}$ School of Public Health, Shanghai Jiao Tong University School of Medicine,

Shanghai, China

${ }^{3}$ Pengpuxincun Community Health Service Center, Shanghai, China

${ }^{4}$ Department of General Practice, Tongji University Affiliated Yangpu Hospital, Shanghai, China

${ }^{5}$ Shanghai General Practice and Community Health Development Research Center, Shanghai, China

${ }^{6}$ Department of Neurosurgery, Navy 971 Hospital, Qingdao, China

${ }^{7}$ Tongji University School of Economics and Management, Shanghai, China

${ }^{8}$ General Practice Center, Southern Medical University Affiliated Nanhai Hospital, Guangzhou, China

${ }^{9}$ Department of Health Service Management, Shanghai Jiao Tong University School of Medicine, Shanghai, China

Contributors NC conceptualised and drafted the manuscript and analysed the data. JS and ZW provided critical review and developed the manuscript. LZ, JH, WY, $\mathrm{CC}, \mathrm{HJ}, \mathrm{XS}, \mathrm{ZY}, \mathrm{QL}$ and YY contributed to data collection. All the authors read and approved the final manuscript. 
Funding This research was supported by the Shanghai Excellent Young Talents Project in Health System (<2018YQ52> (to JS)) and Shanghai Educational Science Research Project $(<\mathrm{C} 2021039>$ (to JS)). Data extraction was funded by the Natural Science Foundation of China $(<71774116>$ (to ZW); $<71603182>$ (to JS)). The analysis and interpretation of the data guided by the statisticians were funded by grants from the National Key R\&D Programme of China $(<2018 Y F C 2000700$, $2018 Y F C 1314700>$ (to ZW)). The writing and revision, including the language improvement, were sponsored by Shanghai Pujiang Programme (<2019PJC072> (to JS) and the Shanghai Public Health Outstanding Young Personnel Training Programme (<GWV-10.2-XD07> (to ZW)).

Competing interests None declared.

Patient consent for publication Not required.

Ethics approval The study was approved by the Ethics Committees of Tongji University (ref: LL-2016-ZRKX-017). None of the inpatients' personal information included in the dataset was available to individuals who did not participate in the research.

Provenance and peer review Not commissioned; externally peer reviewed.

Data availability statement Data are available upon reasonable request. Data may be obtained from a third party and are not publicly available.

Open access This is an open access article distributed in accordance with the Creative Commons Attribution Non Commercial (CC BY-NC 4.0) license, which permits others to distribute, remix, adapt, build upon this work non-commercially, and license their derivative works on different terms, provided the original work is properly cited, appropriate credit is given, any changes made indicated, and the use is non-commercial. See: http://creativecommons.org/licenses/by-nc/4.0/.

\section{ORCID iDs}

Jiaoling Huang http://orcid.org/0000-0003-1975-3937

Zhaoxin Wang http://orcid.org/0000-0002-5022-4497

Jianwei Shi http://orcid.org/0000-0002-3523-6246

\section{REFERENCES}

1 van den Akker M, Buntinx F, Metsemakers JF, et al. Multimorbidity in general practice: prevalence, incidence, and determinants of co-occurring chronic and recurrent diseases. J Clin Epidemiol 1998:51:367-75.

2 Sakib MN, Shooshtari S, St. John P, et al. The prevalence of multimorbidity and associations with lifestyle factors among middleaged Canadians: an analysis of Canadian longitudinal study on aging data. BMC Public Health 2019;19.

3 Calderón-Larrañaga A, Poblador-Plou B, González-Rubio F, et al. Multimorbidity, polypharmacy, referrals, and adverse drug events: are we doing things well? Br J Gen Pract 2012;62:e821-6.

4 Salive ME. Multimorbidity in older adults. Epidemiol Rev 2013;35:75-83

5 Loeppke R, Taitel M, Richling D, et al. Health and productivity as a business strategy. J Occup Environ Med 2007;49:712-21.

6 Nóbrega TCMde, Jaluul O, Machado AN, et al. Quality of life and multimorbidity of elderly outpatients. Clinics 2009;64:45-50.

7 St John PD, Tyas SL, Menec V, et al. Multimorbidity, disability, and mortality in community-dwelling older adults. Can Fam Physician 2014;60:E272-80.

8 Wang L, Si L, Cocker F, et al. A systematic review of cost-ofillness studies of multimorbidity. Appl Health Econ Health Policy 2018;16:15-29.

9 Glynn LG, Valderas JM, Healy P, et al. The prevalence of multimorbidity in primary care and its effect on health care utilization and cost. Fam Pract 2011;28:516-23.

10 Sakib MN, Shooshtari S, St John P, et al. The prevalence of multimorbidity and associations with lifestyle factors among middleaged Canadians: an analysis of Canadian longitudinal study on aging data. BMC Public Health 2019;19:243.

11 Wolff JL, Starfield B, Anderson G, Prevalence AG. Prevalence, expenditures, and complications of multiple chronic conditions in the elderly. Arch Intern Med 2002;162:2269-76.

12 Violán C, Foguet-Boreu Q, Fernández-Bertolín S, et al. Soft clustering using real-world data for the identification of multimorbidity patterns in an elderly population: cross-sectional study in a Mediterranean population. BMJ Open 2019;9:e029594.

13 Ferro MA, Lipman EL, Van Lieshout RJ, et al. Multimorbidity in children and youth across the life-course (my life): protocol of a Canadian prospective study. BMJ Open 2019;9:e034544.
14 Dunbar P, Hall M, Gay JC, et al. Hospital readmission of adolescents and young adults with complex chronic disease. JAMA Netw Open 2019;2:e197613.

15 Sciences TAoM. Multimorbidity: a priority for global health research: the academy of medical sciences, 2018. Available: https://acmedsci. ac.uk/file-download/82222577

16 Wu Y, Wang X, Wu J, et al. Performance of heat-health warning systems in Shanghai evaluated by using local heat-related illness data. Sci Total Environ 2020;715:136883.

17 Shi J, Tang L, Jing L, et al. Disparities in mental health care utilization among inpatients in various types of health institutions: a crosssectional study based on EHR data in Shanghai, China. BMC Public Health 2019;19:1023.

18 Shanghai Statistics Bureau. Shanghai Statistics Yearbook 2018, 2018. Available: https://data.cnki.net/area/yearbook/single/ N2019040068?z=D09

19 HCUP. HCUP chronic condition indicator for the International classification of diseases, 10th revision, clinical modification (ICD-10CM), 2015. Available: www.hcup-us.ahrq.gov/toolssoftware/chronic icd10/chronic icd10.jsp

20 Kaba Özge, Kara M, Odacılar CA, et al. Evaluation of cases of pediatric extrapulmonary tuberculosis: a single center experience. Turk Pediatri Ars 2019;54:86-92.

21 Emeksiz HC, Derinöz O, Akkoyun EB, et al. Age-specific frequencies and characteristics of ovarian cysts in children and adolescents. $J$ Clin Res Pediatr Endocrinol 2017;9:58-62.

22 Barnett K, Mercer SW, Norbury M, et al. Epidemiology of multimorbidity and implications for health care, research, and medical education: a cross-sectional study. Lancet 2012;380:37-43.

23 Russell J, Grant CC, Morton SMB. Multimorbidity in early childhood and socioeconomic disadvantage: findings from a large New Zealand child cohort. Acad Pediatr 2020;20:619-627.

24 Parker SE, Mai CT, Canfield MA, et al. Updated national birth prevalence estimates for selected birth defects in the United States, 2004-2006. Birth Defects Res A Clin Mol Teratol 2010;88:1008-16.

25 Taye M, Afework M, Fantaye W, et al. Congenital anomalies prevalence in Addis Ababa and the Amhara region, Ethiopia: a descriptive cross-sectional study. BMC Pediatr 2019;19.

26 Francine R, Psascale S, Aline H. Congenital anomalies: prevalence and risk factors. Universal J Public Health 2014;2:58-63.

27 Dolk H, Loane M, Garne E. The prevalence of congenital anomalies in Europe. Adv Exp Med Biol 2010;686:349-64.

28 Sallout B, Obedat N, Shakeel F, et al. Prevalence of major congenital anomalies at King Fahad medical City in Saudi Arabia: a tertiary care centre-based study. Ann Saudi Med 2015;35:343-51.

29 Ekwochi U, Asinobi IN, Osuorah DCl, et al. Pattern of congenital anomalies in newborn: a 4-year surveillance of newborns delivered in a tertiary healthcare facility in the south-east Nigeria. $J$ Trop Pediatr 2018;64:304-11.

30 Dutau G, Lavaud F. Allergic rhinitis and its comorbidities. Rev Fr Allergol 2019;59:32-40.

31 Gutierrez MJ, Rodriguez-Martinez C, Nino C, et al. Phenotypical features of obstructive sleep apnea (OSA) in children with chronic rhinitis. Journal of Allergy and Clinical Immunology 2013;131:AB41.

32 Kobayashi R, Miyazaki S, Karaki M, et al. Obstructive sleep apnea in Asian primary school children. Sleep Breath 2014;18:483-9.

33 Shen L, Lin Z, Xu Y, et al. [The relationship between obstructive sleep apnea hypopnea syndrome and adenoid size as well as tonsil size in children]. Lin Chung Er Bi Yan Hou Tou Jing Wai Ke Za Zhi 2014;28:381-5.

34 Sih T, Mion O. Allergic rhinitis in the child and associated comorbidities. Pediatr Allergy Immunol 2010;21:e107-13.

35 Torres AJ. Hemodynamic assessment of atrial septal defects. $J$ Thorac Dis 2018;10:S2882-9.

36 Jain S, Dalvi B. Atrial septal defect with pulmonary hypertension: when/how can we consider closure? J Thorac Dis 2018;10:S2890-8.

37 Abdulkadir M, Abdulkadir Z. A systematic review of trends and patterns of congenital heart disease in children in Nigeria from 1964 2015. Afr Health Sci 2016;16:367-77.

38 Roth TS, Aboulhosn JA. Pulmonary hypertension and congenital heart disease. Cardiol Clin 2016;34:391-400.

39 Cohen JL, Nees SN, Valencia GA, et al. Sildenafil use in children with pulmonary hypertension. J Pediatr 2019;205:29-34.

40 Patel SS, Fernie JC, Taylor AL, et al. Evaluation of predictive models for six minute walk test among children with pulmonary hypertension. Int J Cardiol 2017;227:393-8.

41 Clavé MM, Maeda NY, Thomaz AM, et al. Phosphodiesterase type 5 inhibitors improve microvascular dysfunction markers in pulmonary arterial hypertension associated with congenital heart disease. Congenit Heart Dis 2019;14:246-55. 
42 Hansmann G. Pulmonary hypertension in infants, children, and young adults. J Am Coll Cardiol 2017;69:2551-69.

43 Xi S-B, Wang S-S, Qian M-Y, et al. Predictors of operability in children with severe pulmonary hypertension associated with congenital heart disease. Chin Med J 2019;132:811-8.

44 Huggins JL, Holland MJ, Brunner HI. Organ involvement other than lupus nephritis in childhood-onset systemic lupus erythematosus. Lupus 2016;25:857-63.
45 Levy DM, Kamphuis S. Systemic lupus erythematosus in children and adolescents. Pediatr Clin North Am 2012;59:345-64.

46 Bohmat L, Bessonova I, Shevchenko N. Co-Morbidity index in children with systemic lupus erythematosus. Ann Rheum Dis 2019;78:1988-9.

47 Abad-Díez JM, Calderón-Larrañaga A, Poncel-Falcó A, et al. Age and gender differences in the prevalence and patterns of multimorbidity in the older population. BMC Geriatr 2014;14:75. 